\title{
A Lexical Trunk Approach to the Teaching of English for Science and Technology Reading
}

\author{
Shengxi Jin \\ School of Foreign languages, Changchun University of Science and Technology, Changchun, China \\ Bin Wang \\ School of Foreign Languages, Jilin Agricultural University, Changchun, China
}

\begin{abstract}
Reading is widely accepted as an effective way for EST learners to obtain information and knowledge, but most EST learners in China find that their reading competence is not satisfactory although they have a large size of EST vocabulary and study English grammar well. So how to improve EST learner's reading competence remains a problem urgent to settle for EST teachers and researchers. Lexical trunk approach has been widely applied to teaching written discourse and spoken discourse, and a lot of achievements have been made. And we made investigation into the application of lexical trunk approach to the teaching of EST reading and concluded that lexical trunk approach is very helpful to teaching EST reading. In order to improve EST students' reading competence and enhance the teaching efficiency, teachers should strengthen students' ability to use lexical trunks in EST reading class and it is of critical importance for EST teachers to adopt appropriate strategies and work out scientific class design from the perspective of lexical trunk approach.
\end{abstract}

Index Terms_EST reading, approach, lexical trunk

\section{INTRODUCTION}

With the fast development of science and technology, China is in desperate need of the talents of English for science and technology (hereinafter abbreviated as EST). More and more universities have started to increase the investment into EST teaching. Reading, as one of the most important skills for learners in language learning and acquisition has been attached great importance to by university teachers of English. However, most of the EST students complained that the articles are too complicated and difficult for them to understand although they know the meanings of each word or single sentences. The reasons mainly lie in the fact that teachers usually employ traditional translation approach to the teaching of EST reading, leaving no room for the students to improve their reading competence. Therefore, a more appropriate teaching approach is required to increase the teaching efficiency. Recently, the lexical chunk approach has drawn the attention of many scholars and university teachers of English. With the help of lexical chunks, the second language learners can obtain better accuracy and fluency in language use and strengthen their competence of discourse analysis. This paper attempts to explore how to apply lexical approach to teaching EST reading.

\section{LEXICAL TRUNK APPROACH}

Most researchers have come to a conclusion that lexical chunks are abundant in language, but different definitions have been put forward. Michael Lewis (1997) makes the definition that "lexical items are sanctioned independent units, and they may be individual words socially or full sentences - institutionalized utterances - that convey fixed social or pragmatic meaning within a given community" (p.225). Moon (1997) insists that lexical chunk is "a vocabulary item consisting of a sequence of two words or more (a word is just an orthographic unit)...and it forms a meaningful and inseparable unit" (p.43). Nattinger and DeCarrico (2000) conclude that "lexical chunks are some lexical phrases with varying length, loaded with communicative and discourse functions, and lexical chunk is multi-word lexical phenomena that exist somewhere between the traditional poles of lexicon and syntax, conventionalized form/function composites that occur frequently and have more idiomatically determined meaning"(p.1). Synthesizing the above definitions, we support the definition that lexical chunks are language structures stored in the long-term memory of language learners and they are frequently-occurred, fixed or semi-fixed multi-words or sentences which can be drawn and used automatically rather than being generated and analyzed from grammatical rules. The more lexical chunks stored in the long-term memory of a language learner, the less effort is paid by him to fulfill some language learning tasks.

As for functions of lexical trunks, Nattinger and DeCarrico (2000) divide them into three sorts including social interactions, necessary topics and discourse devices. Social interactional markers are used to describe social relations, consisting conversational maintenance, and conversational purpose. As to necessary topics, those lexical phrases mark topics about which learners are often asked, or ones that are necessary in daily conversations. Discourse devices are lexical phrases that connect the meaning and structure of the discourse. They put forward that all formal categories of lexical phrases are represented in the three functional groups. For teaching purposes, those formal categories can be 
reduced to three, with institutionalized expressions being incorporated into the polyword category, since institutionalized expressions are more or less sentence-length polywords. They employed the three formal categories: polywords, phrasal constraints and sentence builders.

The paper mainly discusses how to apply lexical trunk approach to teaching the reading of EST discourses. Next the characteristics of discourse devices in written texts will be introduced in accordance to what Nattinger and DeCarrico have illustrated in their book entitled Lexical Phrases and Language Teaching. They concluded that the lexical phrases that characterize it reflect this dual purpose because most written discourses are a mix of the interactional and transactional. In written discourses, lexical phrases that predominate are those that function as discourse devices to signal the overall direction and organization of the discourses, but those for necessary topics and conversational purpose also takes place. In signaling the overall direction and organization of the discourse, the discourse devices function to mark high-level transactional information, such as exemplification, relationships between topics, evaluations, qualifications, asides and so on, and indicate its flow through the discourse. Lexical phrases of conversational maintenance are still used to summon the audience before the body of the text begins, as well as to prime and nominate the topic and to close the discourse. Those characteristics of discourse devices are also found in EST discourse. So the next section will be about what EST is and what features EST has.

\section{ENGLISH FOR SCIENCE AND TECHNOLOGY}

English for Science and Technology refers to any piece of written or spoken English dealing with science and technology. As a major subdivision of ESP (English for Special Purpose), EST is a formal linguistic genre which is featured as its objectivity, strictness, and conciseness. Generally, EST discourse distinguishes itself from other types of discourse at lexical, syntactical, and stylistic levels.

Firstly, at the lexical level, three features of EST discourse are summarized as follows: high frequency of nouns, the abundance of compound words, and a large number of terminologies. In order to express the logical and abstract ideas, EST writers often adopt the way verbs and adjectives are nominalized under most circumstances. In some other cases In EST discourse, EST writers also use compound words including compound nouns, compound adjectives and compound verbs to make what they want to express more accurate. EST discourse is used to serve the need of those people in special industrial sections, so terminologies such as captive breeding, meta-analysis are found abundant in medical English texts

Secondly, most EST discourses are found at the syntactical level abundant in wide use of long sentences, of passive voice, of non-predicate verb forms. More long and complex sentences are used in EST discourse. The following example is a long and difficult sentence which is usually designed for the sake of accuracy and faithfulness in describing such logic relationships as reason-result or comparison.

Example One: One might hope that modern brain imaging techniques such as EEG (Electroencephalography), PET(Position Emission Tomography) and fMRI (functional Magnetic Resonance Imaging), which allow activity in different parts of the brain to be measured, might provide a definite resolution to this so-called "state/non-state" argument.

Example Two: Moreover, though researchers have made considerable progress in understanding and predicting the effects of sonic booms, recent studies and community surveys strongly suggest that people find even occasional sonic booms much more disturbing than loud, continuous noise such as that of an airport

In the above examples, the writer uses some interlinear notes, such as EEG (Electroencephalography), PET (Position Emission Tomography) and fMRI (functional Magnetic Resonance Imaging), and clause such as though researchers have made considerable progress in understanding and predicting the effects of sonic booms in order to express very accurate and faithful information although those interlinear notes and attributive clauses make the sentences longer and more complicated.

The passive voice can make sentence structure more compact and the author's intention can be emphasized. One example is as follows.

Example Three: When successfully integrated into the product development cycle, development time can be reduced, and maximum manufacturability and build ability will be achieved.

In the above example, development time, maximum manufacturability and build ability are highlighted as subjects, which make it easy for readers to understand that product development cycle can improve the three items.

Thirdly, the EST discourse style is featured as impersonal description. Very few personal sentences are employed in EST discourse to introduce scientific discoveries and laws because EST discourses mainly aim to introduce the scientific findings, rather than the researchers or discoverers of those scientific achievements. The following is another example:

Example Four: A major part of DFM is the ability to assemble parts easily and free of defects. A key to build-ability is process variation. Dimensional variation management is a structured process for analyzing and managing variation in product design and manufacturing processes to optimize the vehicle build quality. The goal is a robust design that has no critical-build requirements, nor process-control requirements.

In the above example no personal pronouns are used. All the subjects and objects are impersonal, which makes the discourse more objective. Based on the above analysis, we found it very necessary for EST teachers to teach these 
characteristics to students so that they can have a better understanding of EST discourse from perspective of lexical approach.

\section{Application of LeXicAl Trunk Approach to EST ReAding}

Lexical trunk approach has drawn great attention from scholars and researchers and been used in teaching English writing, interpreting and so on. Is it feasible to adopt lexical trunk approach in teaching EST reading? If so, how to teach EST with lexical trunks effectively? In order to answer the above questions, we will, in the following sections, investigate into the role of lexical trunk approach to EST Reading from the perspective of enlarging students' vocabulary in EST reading, improving students' reading speed, and strengthening students' ability to comprehend EST discourse.

\section{A. The Role of Lexical Trunk Approach to EST Reading}

\section{Enlarging learners' vocabulary in EST reading}

Zimmerman (1997) holds that "Vocabulary is central to language and of critical importance to the typical language learner" (p.3). Laufer (1987) points out the importance of vocabulary learning in language acquisition by saying that "the learning of vocabulary lies at the heart of language learning, it would be reasonable to assume that language acquisition studies should devote no less scope and effort to vocabulary than to phonology or grammar." Effective Lexical chunks are stored in learners' memory as completely or partially prefabricated units and it is very easy for EST learners to retrieve them when they are need. Nattinger and DeCarrico (2000) propose that learners can creatively construct sentences and enhance the fluency of language use because phrases are stored and reprocessed as whole chunks in the memory. Lexical chunks are strings of words or a whole sentence which means EST learners can spare fewer efforts to memorize more words and they don't need to memorize word by word in the vocabulary list in an isolated way. In this sense, lexical trunks are considered to form their own contexts with concrete meanings, which make them much easier to remember than those words out of contexts. Through the above analysis, we can conclude that the lexical trunks are very helpful to enlarge EST learners' vocabulary.

2. Improving EST learners' reading speed

In cognitive psychology, memory is a project of critical importance. Memory is the mental capacity to store information, either for short or long periods. Hu (1995) arrives at the conclusion that memory plays a very important role in language learning. To some degree, the language learning process is the process of memory. Therefore, the key point in language learning is to try to memorize what he/she has learned and the important aim of teaching is to teach students how to remember what they have learned successfully and store more information for future use.

Atkinson and Shiffrin (1968), firstly, bring forward a complete and systematic modal of information process. In their model memory can be divided into three phases: sensory register, short-term memory and long-term memory. In the process of EST reading, learners will find it very difficult to transfer what they have read from short-term memory into long-term memory if they have no good approach to enhance their reading efficiency. In that case hindrance becomes inevitable. However, lexical trunk approach can be the remedy for learners to remove the hindrance. Learners are able to construct those single pieces of information into bigger lexical trunks, which can enlarge the processing load in their short-term memory and help to encode rapidly and efficiently the information available in the text. According to the effect of chunking on memory, the information encoded is easy to retrieve and decoded when necessary. Therefore, if EST students read through the lexical chunk approach instead of word-by-word approach, their speed to decode the text will be increased. Here is the conclusion that lexical chunk approach can improve EST students reading speed.

3. Strengthening learners' ability to comprehend EST discourse

Lexical trunks are form/function composites and supply rich semantic and contextual information, which help EST learners to comprehend EST discourse. Most students cannot accurately comprehend EST discourses although they have already grasped a large number of words and phrases. The main reason lies in their neglect of the importance of the cohesion and coherence in the understanding of EST discourses. However, lexical trunks are parts of language that often have clearly defined role in guiding the overall discourse. In particular, they are the primary markers which signal the direction of discourse (Nattinger, 1986). When they serve as discourse devices, they serve specific pragmatic functions, which contribute to aiding learners to construct quickly the semantic connections between sentences. Meanwhile learners can make accurate prediction as for the author's intention through what has been activated by lexical trunks. An example is as follows.

Example Five: Like a lot of things, we think that achieving artificial intelligence is harder than it looks. In the 1950s, the pioneers of AI confidently predicted that, $\underline{b}$ the end of the century, computers would be conversing with us at work and robots would be performing our housework. But it is concluded that as useful as computers are, they are nowhere close to achieving anything remotely resembling these early aspirations for humanlike behavior.

In the above text, three chunks are underlined. We think is the topic shifter used to express the authors' viewpoints; by the end of is temporal connector highlighting the time; it is concluded that is functioning as a summarizer which sums up the authors' points of view. Grasping the features of those trunks, learners can make it easy to know the discourse structure clearly and then understand the authors' purpose. To sum up the above arguments, it can be inferred that lexical trunks play an important role in strengthening learners' ability to grasp the textual structure of EST. 


\section{B. Teaching EST Reading with Lexical Trunks}

1. Cultivating learners' awareness of lexical trunks in EST

Lewis (1997) suggests that conscious awareness of what constitute a possible chunk provides learners with tool that enables them to process input more effectively. However, most EST students are poor in the knowledge of lexical trunks and haven't realized the important role that lexical trunks play in EST reading. Nattinger \& DeCarrico (2000) holds that successful readers tend to skip words they view as unimportant to total phrase meaning. While unsuccessful readers seldom skip any, viewing all words as equally important in their contribution to the total meaning. Less proficient readers, such as L2 readers tend to focus on reading as decoding process rather than as a meaning-getting process, and depend on local strategies, having to do with sound-letter, word-meaning, sentence syntax, and text details.

Therefore more efforts and attention of teachers and learners should be paid to lexical trunks in EST reading. Firstly it is very necessary for EST teachers to instruct students to know what lexical trunks are and its classification and function in learning EST. Then EST learners should be taught to grasp some number of lexical trunks frequently used. In teaching EST reading, teachers should use more lexical trunks to express their ideas and the main contents of EST texts so that students can have more opportunities to acquire those trunks. More contexts are required to explain those lexical trunks in EST texts. Gradually the lexical trunk size of EST learners could be enlarged. Next EST teachers should focus on improving students' ability to use these lexical trunks in reading EST discourse or completing different learning tasks. To realize this goal, teachers need to adopt effective teaching strategies in EST class, which will be expanded on in the following section.

2. Adopting effective strategies in EST class

Nattinger \& DeCarrico (2000) also suggest that "Teachers need provide strategies to assist students in synthesizing meaning in larger segments of text and it is useful for teachers to teach students to read with lexical chunks"(p.160). During the period of teaching EST reading, students should be acquainted with some reading strategies relating lexical trunks. For example, EST students can be taught how to grasp the writer's intention through lexical trunks or how to know the organizational mode of EST discourse through lexical phrase markers.

Brown and Payne (1994) have conducted an analysis that resulted in a very clear model for vocabulary learning, which fell into five essential steps: (1) having sources for encountering new words, (2) getting a clear image, either visual or auditory or both, for the forms of the new words, (3) learning the meaning of the words, (4) making a strong memory connection between the forms and meanings of the new words and (5) using the words. Based on the above steps, EST teachers should help students to identify lexical trunks by designing some learning tasks such as underlining the lexical trunks in the given text. After underling the trunks, students can adopt the way of cooperative study by exchanging what they have identified with their team members, through which their interest in lexical trunks could be aroused. Then teachers give the right answers and make further explanation relating those lexical trunks. Meanwhile EST teachers should help students to make further analysis into those trunks in the reading materials so that they can comprehend the text correctly. Hulstijn et al (1996) propose that increasing the repetition of the new words is a good way to acquire them. So teachers can give dictation to students and ask them to make sentences by using those trunks in order to strengthen students' ability to grasp those lexical phrases.

Lautamatti (1987) identifies three possible progressions that result in cohesive discourse and students have t follow three procedures in performing a topical structural analysis: they must identify sentence topics, then determine sentence progression, and finally chart the progress of sentence topics. In EST discourse, surrounding lexical phrases can signal cohesive patterns of co-ordination among sentences. Once students become adept at identifying sentence level cohesion and signaling cues, they will apply the same principles across sentence boundaries and begin to focus on the global cues and questions that lend coherence to discourse. To highlight these cues, EST teachers can design exercises for isolating lexical phrases in academic lectures. Reading activities are important for learning such lexical phrases. Texts must be selected and modified to expose students to the most frequent of these phrases or to those that are the center of a particular lesson (Nattinger \& DeCarrico, 2000). So another very effective strategy is to design filling blanks task for EST learners. The following is one of the filling-blank examples.

\section{Example Six:}

Use those lexical phrases to fill in the EST passage

carry out, but, such as, be based on, no more than, a limited area, sometimes, one's ability to, even if, in the quest to

Computers can imitate thought processes those necessary to some complex tasks very logically. They can manipulate symbols extremely fast and possess great memories.___ do they have intelligence? Hurbert Dreyfus, professor of philosophy at the University of California at Berkeley, remarked that a computer is still __ a mechanized idiot, i.e., a dumb machine that exhibits a remarkable skill in _. What "intelligence" computers do have is__ recognize patterns and solve problems using programmed logic. They cannot reason, but can only make educated guesses; they cannot generalize. solutions the human attribute called common sense, which may not always be logical.

Another problem is that we define artificial intelligence as "the ability of computers to think and reason like the human mind: the ability to use common sense, intuition, judgment and evaluation," scientists have not determined exactly how the mind does this. Human intelligence is not entirely understood, and scientists are just scratching the 
surface __ discover how humans get ideas and how they think.

This kind of task can strengthen students' ability to use those lexical trunks and is very useful for them to have a general view of the short EST passage. Based on the above passage, teachers should help students analyze the function of those trunks such as discourse devices, topic makers, organizing functions and so on. Through analysis students will find it easier to comprehend the passage.

In addition to the strategies discussed, lexical trunks translation or rewriting is worth adopting. It is of great importance for teachers to integrate those strategies and devise effective class design so that the teaching of EST reading can be conducted reasonably and efficiently.

\section{CONCLUSiON}

Based on the above analysis, it can be concluded that lexical trunk approach has a positive effect on the teaching and learning of EST reading. Lexical trunk approach is propitious to enlarging EST learners' vocabulary size, improving their reading fluency and strengthening their reading competence. In EST reading class, teachers are supposed to cultivate students' conscious awareness of lexical trunks in EST discourse and encourage them to apply lexical trunk strategy to EST reading. Meanwhile it is also critical for teachers to work out feasible class design in order to carry out effectively the lexical trunk approach in teaching EST reading.

Note:

This paper is a phase research result of "A Study of the Basic Problems in Sino-America Science and Technology Exchange", a Fund Project of Social Sciences of Jilin Province in 2010, Project No.: 2010B30

\section{REFERENCES}

[1] Atkinson, R.C. \& Shiffrin, R.M. (1968). Human Memory: A proposed system and its control processes. New York: Academic Press.

[2] Brown, C., \& M. E. Payne. (1994). Five Essential Steps or Processes in Vocabulary Learning. Paper presented at the TESOL 1994, Baltimore, Maryland, USA.

[3] Fan Yingfang, Yang Qi \& Yang Zhenhua. (2010). EST Reading Course. Haerbin: Haerbin Institute of Technology Press.

[4] Hu Chundong. (1995). English-teaching Methodology. Bei Jing: Higher Education press.

[5] Hulstijin et al (1996). Incidental Vocabulary Learning by Advanced Foreign Language Students: the influence of marginal glosses, dictionary use, and reoccurrence of unknown words. Modern Language Journal 80: 327.

[6] Kong Qingyan \& Zhang Xu. (2000). New EST Reading Course. Dalian: Dalian University of Technology Press.

[7] Laufer, B. (1987). Teaching Vocabulary: The lexical perspective of reading comprehension. English Teaching Journal (35)59-67.

[8] Lautamatti, L. (1987). Observations on the development of the topic in simplified discourse in U. Connor and R. Kaplan(eds.) Writing across Languages : Analysis of L2 text. Reading, Mass.: Addison-Wesley.

[9] Lewis, M. (1994). A Lexical Approach. London: Cambridge University Press

[10] Lewis, M. (1997). Pedagogical Implications of the Lexical Approach. Cambridge: Cambridge University Press.

[11] Moon, R. (1997). Vocabulary Connections: Multi-word Items in English. In Norbert Schmitt and Michael J. McCarthy (eds). Vocabulary: Description, Acquisition, and Pedagogy. Cambridge: Cambridge University Press.

[12] Nattinger. James (1986). Lexical phrases, functions and vocabulary acquisition. The ORTESOL Journal. 7:1-14.

[13] Nattinger. James \& DeCarrico Jeanette. (2000). Lexical Phrases and Language Teaching. Shanghai: Shanghai Foreign Language Education Press.

[14] Zimmerman, C.B. (1997). Historical trends in second language vocabulary instruction Coady, J. \& Huckin, T. Second Language Vocabulary Acquisition. Cambridge: Cambridge University Press.

Shengxi Jin was born in Dehui, China in 1979. He received his M.A degree in linguistics from Jilin University, China in 2007. He is currently a lecturer in the School of Foreign Languages, Changchun University of Science and Technology. His research interests include cognitive linguistics, pragmatics and business English teaching.

Bin Wang was born in Changchun, China in 1979. She received her M.A degree in linguistics from Jilin University, China in 2006. She is currently a lecturer in the School of Foreign Languages, Jilin Agricultural University. Her research interests include cognitive linguistics, applied linguistics. 